\title{
Untersuchungen über die Enzyme von Bombyx mori L. VI. Mitteilung.
}

Über die Blutlipase.

$$
\text { Von }
$$

Kazuo YamafoJI.

\author{
(Aus dem Biochem. Insiliut der Landw. Ableilung \\ der Kaiserl. Kyushu-Univ. su Fukuoka, Japan.)
}

(Eingegangen am 5, Januar 1934)

Der erste Versuch zu einer quantitativen Bestimmung der Blutlipase bei höheren Tieren findet sich in der Arbeit von Rona und Michaelis ${ }^{(1)}$, die die Spaltung von Mono- oder Tributyrin durch das Ferment mit der stalagmometrischen Methode verfolgten.

Was Insekten betrifft, so ist vielleicht zum erstenmal vom Verf. ein solcher Versuch gemacht worden und im nachstehenden möchte er die Ergebnisse seiner Untersuchungen über die Blutlipase von Bombyx mori mitteilen.

1) Methodik:- Wenn nicht anders angegeben, wurden $25 \mathrm{ccm}$ gesättigte wässerige Tributyrinlösung, $2.5 \mathrm{ccm} \mathrm{M} / 3$ Phosphatpufferlösung von $\mathrm{Ph} 6.8$ und $0.5 \mathrm{ccm}$ Blut vermischt. Je nach 0,10,20 und 30 Minuten Erwärmung bei $20^{\circ}$ wurde die Abspaltung von Tributyrin mit dem Stalagmometer ermittelt. Die Wirksamkeit der Blutlipase wurde mit den Konstanten, die nach der Gleichung der monomolekularen Reaktion berechnet wurden, verglichen.

2) Optimale $P h$.

\begin{tabular}{c|c|c|c|c|c}
\hline \multicolumn{2}{c|}{ Larve, o } & \multicolumn{2}{c|}{ Larve, o } & \multicolumn{2}{c}{ Puppe, o } \\
\hline $\mathrm{Ph}$ & $\mathrm{k} .10^{2}$ & $\mathrm{Ph}$ & $\mathrm{k} .10^{2}$ & $\mathrm{Ih}$ & $\mathrm{k} .10^{2}$ \\
\hline 5.3 & 0.48 & 6.5 & 0.53 & 7.0 & 0.83 \\
6.2 & 0.67 & 6.8 & 0.98 & 7.4 & 0.85 \\
7.0 & 1.20 & 7.2 & 1.12 & 7.7 & 0.96 \\
$\mathbf{8 . 0}$ & $\mathbf{1 . 1 8}$ & 7.7 & $\mathbf{1 . 4 9}$ & 8.0 & 0.73 \\
\hline
\end{tabular}

3) Optimaltemperatur.

\begin{tabular}{c|c|c|c}
\hline \multicolumn{2}{c|}{ Larve, P, $\mathrm{Ph}=6.8$} & \multicolumn{2}{c}{ Puppe, $8, \mathrm{Ph}=7.7$} \\
\hline Temp. & $\mathrm{k} .10^{\mathrm{2}}$ & Temp. & $\mathrm{k} .10^{2}$ \\
\hline 5 & 0.56 & 20 & 0.33 \\
20 & 1.23 & 30 & 0.77 \\
35 & 3.46 & 40 & 1.16 \\
50 & 3.07 & 50 & 1.05 \\
\hline
\end{tabular}


4) Kinetil.

Bei Puppe : $50 \mathrm{ccm}$ gesätt. Tributyrinlösung $+1.5 \mathrm{ccm}$ Pufferlösung $+0.5 \mathrm{ccm}$ Blut, $\mathrm{Ph}=7.7, \quad$ Temp, $=20^{\circ}$

\begin{tabular}{c|c|c|c|c}
\hline \multirow{2}{*}{ Zeit, Minuten } & \multicolumn{2}{|c|}{ Larve, \% $\mathrm{Ph}=6.8$} & \multicolumn{2}{|c}{ Puppe,, $\mathrm{Ph}=7.7$} \\
\cline { 2 - 4 } & $\mathrm{a}-\mathrm{x}$ & $\mathrm{k} .10^{2}$ & $\mathrm{a}-\mathrm{x}$ & $\mathrm{k.10^{2 }}$ \\
\hline 0 & 73 & - & 73 & - \\
10 & 47 & 1.91 & 60 & 0.85 \\
20 & 31 & 1.86 & 50 & 0.82 \\
30 & 20 & 1.87 & 41 & 0.84 \\
40 & - & - & 34 & 0.83 \\
50 & - & - & 28 & 0.83 \\
60 & - & - & 24 & 0.81 \\
\hline
\end{tabular}

$a-x$ bedeutet die in der Zeit $t$ noch vorhandene Menge der Tributyrins (gesättigte wässerige Lösung $\mathbf{= 1 0 0}$ ).

\begin{tabular}{c|c|c|c}
\hline \multicolumn{2}{c|}{ Puppe, ㅇ, $\mathrm{Ph}=6.8$} & \multicolumn{2}{c}{ Puppe, $\delta, \mathrm{Ph}=7.7$} \\
\hline Fermentmenge, ccm & $\mathrm{k} .10^{2}$ & Fermentmenge, ccm & $\mathrm{k.10^{2 }}$ \\
\hline 0.25 & 0.47 & 0.20 & 0.48 \\
0.50 & 0.88 & 0.40 & 0.91 \\
\hline
\end{tabular}

5) Einfuss von Chinin oder Atoxyl.

$2 \mathrm{ccm}$ Pufferlösung $+0.5 \mathrm{ccm}$ Blut $+1 \mathrm{ccm}$ Giftlösung. Nach $30 \mathrm{Min}$. Zugabe von $50 \mathrm{ccm}$ Tributyrinlösung.

\begin{tabular}{c|c|c|c||c|c|c|c}
\hline \multicolumn{2}{c|}{ \%, $\mathrm{Ph}=7.7$} & \multicolumn{2}{c||}{$\delta, \mathrm{Ph}=6.8$} & \multicolumn{2}{c|}{ o, $\mathrm{Ph}=7.7$} & \multicolumn{2}{c}{ o, $\mathrm{Ph}=6.8$} \\
\cline { 1 - 7 } $\begin{array}{c}\text { Chinin, } \\
\mathrm{mg}\end{array}$ & $\mathrm{k.10^{2 }}$ & $\begin{array}{c}\text { Chinin, } \\
\mathrm{mg}\end{array}$ & $\mathrm{k.10^{2 }}$ & $\begin{array}{c}\text { Atoxyl, } \\
\mathrm{mg}\end{array}$ & $\mathrm{k} .10^{2}$ & $\begin{array}{c}\text { Atoxyl. } \\
\text { mg }\end{array}$ & $\mathrm{k} .10^{2}$ \\
\hline 0 & 0.79 & 0 & 0.72 & 0 & 0.43 & 0 & 0.95 \\
5.0 & 0.58 & 2.5 & 0.64 & 5.0 & 0.38 & 2.5 & 0.64 \\
10.0 & 0.41 & 5.0 & 0.53 & 10.0 & 0.36 & 5.0 & 0.55 \\
\hline
\end{tabular}

6) Unterschied zwischen gut gewachsenen und schlecht gewachsenen Raupen.

\begin{tabular}{|c|c|c|c|c|}
\hline & \multicolumn{2}{|c|}{ Gut gewachs, Raupen } & \multicolumn{2}{|c|}{ Schlecht gew. Raupen } \\
\hline & ㅇ & $\hat{\delta}$ & q & $\therefore \delta$ \\
\hline $\mathrm{k} .10^{2}$ & 1.11 & 0.99 & 1.14 & 1.06 \\
\hline
\end{tabular}


7) Änderungen durch Hunger bei der Larve.

\begin{tabular}{c|c|c|c|c}
\hline & Hungerstund. & 1 & 23 & 47 \\
\hline \multirow{2}{*}{$k .10^{\circ}$} & $\circ$ & 0.72 & 0.84 & 1.27 \\
& $\delta$ & 1.09 & 1.06 & 1.40 \\
\hline
\end{tabular}

8) Unterschied zwischen gesunden und kranken Raupen.

\begin{tabular}{c|c|c|c|c}
\hline \multirow{2}{*}{} & \multicolumn{2}{|c|}{ Gesunde Raupen } & \multicolumn{2}{c}{ Krank. Raup. (Nankabyo) } \\
\cline { 2 - 5 } & $\circ$ & $\hat{\circ}$ & $\circ$ & $\hat{\circ}$ \\
\hline $\mathrm{k} . \mathbf{1 0}^{2}$ & 1.52 & 1.06 & 1.58 & 1.36 \\
\hline
\end{tabular}
mori.

9) Veränderungen im Laufe der drei Entwicklungsperioden ron Bombyx

\begin{tabular}{|c|c|c|c|}
\hline & & \multicolumn{2}{|c|}{ k.10 $0^{3}$} \\
\hline & Tage & 우 & $\hat{b}$ \\
\hline \multirow{3}{*}{ Larve } & $\int^{3}$ & 0.86 & 0.93 \\
\hline & V. Lebensalter $\{6$ & 0.72 & 1.09 \\
\hline & 18 & 1.14 & 1.15 \\
\hline \multirow{2}{*}{$\begin{array}{c}\text { Einspinnen des } \\
\text { Kokons }\end{array}$} & 2 & 1.89 & 2.19 \\
\hline & 5 & 2.00 & 1.76 \\
\hline \multirow{4}{*}{ Puppe } & 1 & 1.70 & 1.71 \\
\hline & 3 & 1.41 & 1.55 \\
\hline & 7 & 0.95 & 1.18 \\
\hline & 12 & 0.82 & 0.60 \\
\hline Schmetterling & 1 & 0.79 & - \\
\hline
\end{tabular}

10) Diskussion der Versuchsresultate.

Wie oben erwähnt, habe ich in meiner vorliegenden Arbeit über einige Eigenschaften der Blutlipase von Bombyx mori geforscht und aufgeklärt, dass diese Lipase in ihrer enzymatischen Natur derjenigen des Blutes höherer Tiere sehr nahe steht.

Aber über die Herkunft der Blutlipase bei der Seidenraupe können wir wie bei höheren Tieren nichts sagen, denn wir haben keine eingehenden Angaben über die Organlipasen bei dièsem Insekt.

Darauffolgend kommt die Frage der biologischen Bedeutung der Blutlipase.

Bei den höheren Tieren scheint die Blutlipase nichts mit der normalen 
Ernährung $z u$ tun $z u$ haben. Aber in der fünften Mitteilung ${ }^{(2)}$ habe ich bereits über die engen Beziehungen zwischen den Blutdisaccharasen bei der Seidenraupe und den chemischen Umsetzungen der Disacharosen im Körper derselben gesprochen. Diese Beziehung der Blutfermente von Bombyx mori zum Stoffwechsel behalten auch für die Blutlipase ihre Geltung.

Das Maulbeerblatt enthält kein echtes Fett, dagegen die Seidenraupe immer beträchtliche Mengen Körperfett aufzuweisen hat. Im Digestionssafte der Seidenraupe kommt keine oder nur wenig Lipase vor, im Blut derselben indessen eine verhältnismässig wirksame Lipase. Bei Hunger ist die Blutlipase vermehrt. Die Lipase der kranken Raupe ist wirksamer als die der gesunden. Eine erhebliche Anlagerung des Körperfetts geschieht im fünften Lebensalter der Larve, Die Puppe und der Schmetterling leben hauptsächlich auf eigene Kosten des Körperfettes. Im Laufe von drei Entwicklungsperioden von Bombyx mori wirkt die Blutlipase in den Tagen des Kokonspinnens und des Verpuppens am aktivesten.

Aus den oben angeführten Data geht hervor, dass die Blutlipase bei Bombyx mori in der Assimilation oder beim Abbau des Fetts eine wesentliche Rolle spielt.

\section{Zusammenfassung.}

(1) Die Spaltung des Tributyrins durch die Blutlipase von Bombyx mori kann in guter Annäherung mit Hilfe der Gleichung der monomolekularen Reaktion ausgedrückt werden.

(2) Die Blutlipase zeigt die optimale Aktivität bei Ph 7.7 ; ihr Temperaturoptimum liegt bei $40^{\circ}$.

(3) Die Lipase ist gegen Chinin oder Atoxyl wenig empfindlich.

(4) Die Wirkung der Lipase ist bei kranken Raupen (Nankabyosan) viel höher als bei gesunden und auch bei schlecht gewachsenen Raupen etwas stärker als bei gut gewachsenen. Durch Hunger wird sie gesteigert.

(5) Die Blutlipase wird im fünften Lebensalter der Larve mit dem Wachstum stärker, die ihr Maximum in den Tagen des Kokonspinnens und des Verpuppens erreiclit und dann ist sie in den Perioden der Puppenruhe und des Schmetterlingslebens allmählich vermindert. Es besteht kein wesentlicher Geschlechtsunterschied in der Lipasewirksamkeit.

(6) Bei Bombyx mori scheint die Blutlipase am Fettstoffwechsel Anteil zu nehmen.

Zum Schluss danke ich herzlich Herrn Prof. Y. Okuda für seine Anregung zu diesen drei Arbeiten.

\section{Literatur}

(1) P. Rona u. L. Michaelis: Biochem. Z., 31, 435, (1911).

(2) K. Yamafuji: Bull. Agr. Chem. Soc. Japan, 10, 19, (1934). 